\title{
EDITORIAL
}

\section{Should ICU clinicians follow patients after ICU discharge? No}

\author{
Bharath Kumar Tirupakuzhi Vijayaraghavan ${ }^{1}$, Xavier Willaert ${ }^{2}$ and Brian H. Cuthbertson ${ }^{3^{*}}$
}

๑ 2018 Springer-Verlag GmbH Germany, part of Springer Nature and ESICM

\section{Introduction}

Post-intensive care syndrome (PICS) describes new or worsening impairments of physical, cognitive or mental health resulting from an episode of critical illness and its treatment and lasting after discharge from the intensive care unit (ICU) [1]. The incidence of PICS varies based on the domain impacted, ranging from up to $25 \%$ for physical and cognitive and up to $60 \%$ for psychiatric disturbances $[2,3]$. Even as we continue to make significant impact on mortality, short- and long-term consequences of survivorship are emerging as the new frontier.

\section{Why we should not follow up critically ill patients after ICU discharge}

As the definition of PICS suggests, the extent of impairment spans several domains. Although critical illness seems to be the common pathway leading to these disturbances, treatment of ICU survivors with these impairments needs expertise from a wide array of clinical teams. These may include physiotherapists and occupational therapists, psychiatrists, geriatricians, neurologists and rehabilitation experts. The intensive care physician's knowledge and skills, albeit broad, do not reliably extend to any of these domains even though our advocacy certainly extends there. The advent of specialized medicine stems from the recognition that medicine is

*Correspondence: brian.cuthbertson@sunnybrook.ca

${ }^{3}$ Department of Critical Care Medicine, Sunnybrook Health Sciences Centre, University of Toronto, 2075 Bayview Avenue, Room D108, Toronto, ON, Canada

Full author information is available at the end of the article

For a contrasting viewpoint, please go to https://doi.org/10.1007/s0013 4-018-5260-3. too expansive for any one group to claim expertise over all domains. PICS should be no different, in that it needs to be treated by dedicated practitioners with expertise in these specific domains.

Second, we also believe that any time spent practicing outside of the ICU takes us away from our core area of practice. In the current model, many intensive care physicians already practice in their base specialty; the addition of ICU follow-up could have important implications in terms of knowledge and skill attrition as well as opportunity costs on our time.

Finally, it is also clear from the high-quality randomized controlled trials (see below) that well-intentioned, existing interventions, designed and delivered by ICU teams after ICU discharge, have not yielded the desired results. So maybe it's time to let more expert clinicians take over the helm!

\section{The evidence}

A number of ICU and post-ICU interventions have been evaluated with disappointing results to date. Cuthbertson and colleagues conducted a randomized evaluation [4] of a nurse-led intervention on improving post-ICU quality of life on 286 patients after ICU admission. The intervention involved a nurse-led complex intervention including a manual-based, self-directed, physical rehabilitation program developed by physiotherapists and directed by a study nurse with medical oversight. Patients monitored their own progress and were reviewed by nurses at 3 and 9 months after discharge. The primary outcome of the health-related quality of life (HRQoL) score at 12 months showed no difference between the groups. There was no improvement in any of the secondary outcomes either.

Walsh and colleagues [5] studied the effect of a strategy of hospital-based physical rehabilitation and information provision after ICU discharge in a randomized clinical trial on 240 critically ill patients. The 


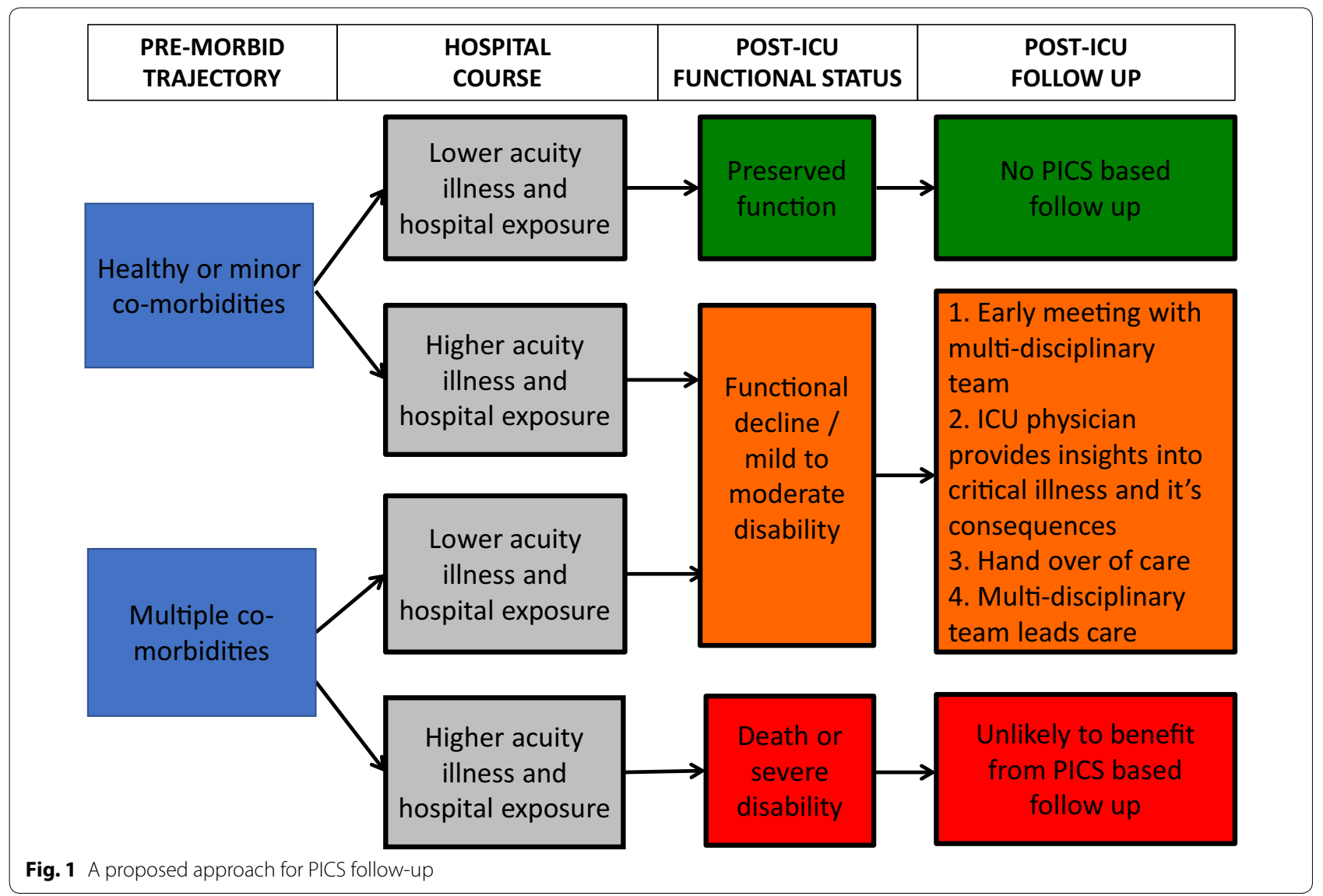

intervention involved rehabilitation that increased the frequency of mobility and exercise therapies, increased dietetic assessment and treatment, used individualized goal setting and provided greater illness specific information. In this study, the primary outcome measure was similar between the groups. The study also found no difference in the HRQoL, anxiety and depressive symptoms, post-traumatic symptoms or any of the physical symptom scores between groups at 3,6 or 12 months.

Similarly, other RCTs of post-ICU interventions [6-9] have failed to show improvement in functional outcomes. There are several reasons why these interventions may have been ineffective including the complexity of the pathophysiology, a focus on physical rehabilitation alone, inability to identify and target high-risk groups, inability to individualize therapy, a focus on the wrong outcome measures and a lack of input from other expert providers such as therapists, neurologists, psychiatrists, geriatricians and rehabilitation physicians. These challenges seem to speak to why follow-up interventions led by intensive care teams are unlikely to be effective in the future.

\section{Where can we help then?}

We identify two specific areas where intensive care physicians can make a meaningful impact on outcomes after ICU discharge:

1. Identify high-risk groups for the development of PICS (e.g., high severity of illness, frailty, prior cognitive impairment, pre-existing disability, etc.) and focus on mitigating these stressors with interventions delivered within the ICU (prevention/treatment of delirium, early mobilization, use of the $\mathrm{ABCDE}$ bundle [10], ICU diaries [11]).

2. Facilitate post-ICU care-we propose triaging ICU survivors into three broad groups (green, orange and red categories) (Fig. 1) depending on their comorbidity and acute severity of illness. We suggest scheduling a review for all "orange" patients after their ICU/ hospital discharge with the previously identified care providers for a needs assessment where this multidisciplinary team can develop an individualized followup plan. The intensive care physician at this meeting can provide important perspectives to the patient and other team members regarding the critical illness 
events and their potential longer-term consequences. They would then handover their patient to this team's care. Note that we do not propose such follow up for "red" or "green" patients as we believe that neither of these groups will sufficiently benefit from this model of care. We accept that current methods to identify "orange" patients are inadequate (see below).

\section{Future research}

Given the lack of benefit for these post-ICU strategies, we may need to rethink our approach to improving PICSrelated outcomes. Future trials should evaluate the role of multidisciplinary and interprofessional team-based programs with ICU physicians playing the role of facilitators. Precisely identifying the "orange" patients who can benefit will be an important part of this agenda. Research must also continue to focus on biologic mechanisms that contribute to PICS, which in turn can guide further interventional research.

In conclusion, while we recognize the desire of the intensive care physician to contribute to helping patients after critical illness, intensivist-led follow-up programs do not seem to be effective, and therefore we should limit ourselves to supplying information to our patients and to multiprofessional teams that are better equipped to help them with their ongoing challenges.

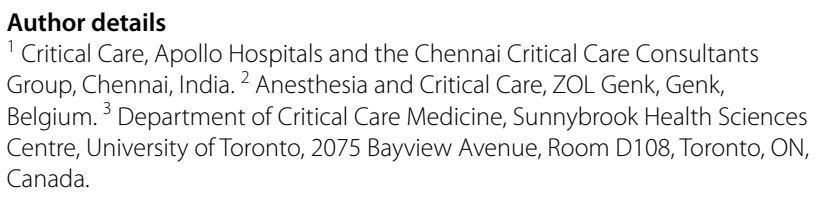

\section{Funding}

None.

\section{Compliance with ethical standards}

\section{Conflicts of interest}

The authors declare that they have no conflict of interest.
Received: 2 February 2018 Accepted: 22 February 2018

Published online: 27 July 2018

\section{References}

1. Needham DM, Davidson J, Cohen H, Hopkins OR, Weinert C, Wunsch $\mathrm{H}$ et al (2012) Improving long-term outcomes after discharge from intensive care unit: report from a stakeholders' conference. Crit Care Med 40(2):502-509

2. Rawal G, Yadav S, Kumar R (2017) Post-intensive care syndrome: an overview. J Transl Int Med 5(2):90-92

3. Desai S, Law T, Needham DM (2011) Long-term complications of critical care. Crit Care Med 39(2):371-379

4. Cuthbertson BH, Rattray J, Campbell MK, Gager M, Roughton S, Smith A et al (2009) The PRaCTICaL study of nurse led, intensive care follow-up programmes for improving long term outcomes from critical illness: a pragmatic randomised controlled trial. BMJ 339:b3723

5. Walsh TS, Salisbury LG, Merriweather JL, Boyd JA, Griffith DM, Huby G et al (2015) Increased hospital-based physical rehabilitation and information provision after intensive care unit discharge. the recover randomized clinical trial. JAMA Intern Med 175(6):901-910

6. Elliott D, McKinley S, Alison J, Aitken LM, King M, Leslie GD (2011) Healthrelated quality of life and physical recovery after critical illness: a multicentre randomised controlled trial of a home-based physical rehabilitation program. Crit Care 15:R142

7. Jensen JF, Egerod I, Bestle MH, Christensen DF, Elklit A, Hansen RL et al (2016) A recovery program to improve quality of life, sense of coherence and psychological health in ICU survivors: a multicentre randomized controlled trial, the RAPIT study. Intensive Care Med 42:1733-1743

8. Morris PE, Berry MJ, Files C, Thompson C, Hauser J, Flores L et al (2016) Standardized rehabilitation and hospital length of stay among patients with acute respiratory failure: a randomized clinical trial. JAMA 315(24):2694-2702

9. Moss M, Nordon-Croft A, Malone D, Van Pelt D, Frankel SK, Warner ML et al (2016) A randomized trial of an intensive physical therapy program for patients with acute respiratory failure. Am J Respir Crit Care Med 193(10):1101-1110

10. Balas MC, Vasilevskis EE, Olsen KM, Schmid KK, Shostrom V, Cohen MZ et al (2014) Effectiveness and safety of the awakening and breathing coordination, delirium monitoring/management and early exercise/ mobility bundle. Crit Care Med 42(5):1024-1036

11. Jones C, Backman C, Capuzzo M, Egerod I, Flaatten H, Granja C et al (2010) Intensive care diaries reduce new onset post traumatic stress disorder following critical illness: a randomised, controlled trial. Crit Care 14(5):R168 